\title{
¿QUÉ APORTA LA FENOMENOLOGÍA A LA RELACIÓN ENFERMERA-PACIENTE?
}

\author{
LUIS CIBANAL JUAN - Prof. Titular de Enfermería en Salud Mental \\ Mª Carmen Arce Sánchez - Psicóloga. \\ Dpto. Enfermería Universidad de Alicante \\ Apartado Correos 99. 03080-Alicante.
}

\section{RESUMEN}

En este artículo se pretenden exponer las bases filosóficas que subyacen en la relación enfermera-paciente. La relación enfermera-paciente, tal y como generalmente se viene estableciendo está basada en la concepción humanista y fenomenológica. En este trabajo se parten de los principios aportados por Husserl, dejando un tanto al margen las aportaciones del existencialismo francés. La tesis central de este estudio se puede resumir en una afirmación: la concepción fenomenológica nos confirma la visión holística que debe desarrollar la enfermera al abordar al paciente.

\section{WHAT DOES PHENOMENOLOGY CONTRIBUTE TO THE NURSE-PATIENT RELATIONSHIP?}

\section{SUMMARY}

The pre-supposed philosophical basis for the nurse-patient relationship is considered in the present article. The relationship between a nurse and a patient, as has been generally established, is seen from a humanistic and phenomenological perspective. Our study, based on Husserl's point of view - besides that of the French existencialist movement - confirms our main point: the patient should be considered from an holistic approach.

\section{a) Introducción}

En todos los tiempos ha existido en medicina dos formas paralelas de abordar la persona:

Una concebía el hombre como un todo, viviente; por tanto la enfermedad y todo objetivo terapéutico estaba incluido en una relación de globalidad. (Ejemplo: la medicina humoral de Hipócrates). La otra, concebía cada parte del cuerpo por separado, dando preferencia así a una concepción analítica,

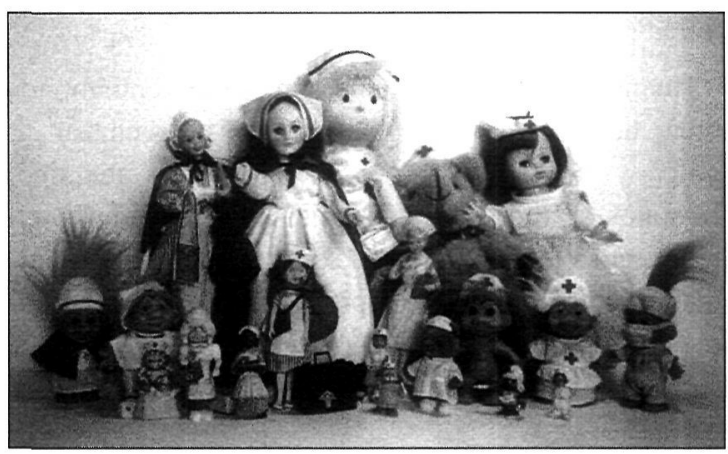


basada en las relaciones causales, "científicamente demostrables", de la enfermedad de la persona. Esta última visión, que nos permite un estudio científico mucho más "fácil" de la relación terapéutica, ha invadido prácticamente todo el campo de la medicina, y también de la enfermería. Nosotros queremos señalar de alguna manera los límites que esto entraña en la relación del enfermero/a con el paciente. No se trata de renunciar a todo lo que esta concepción nos ha aportado de utilidad. El problema nos parece más bien tratar de hacer una síntesis, con un espíritu pragmático y útil, de ver lo que una nos ha aportado y lo que la otra puede aún aportarnos.

\section{b) Principios del proceso causal y deductivo.}

Nuestra cultura occidental está impregnada del razonamiento causal...(causa-efecto) sin darnos cuenta muchas veces. Estamos acostumbrados a tener presente en nuestro espíritu un esquema que permite relacionar cuidadosamente todo acontecimiento presente con unas consecuencias en el futuro, y por tanto creemos, que podemos determinar las causas para así de alguna manera prever, suprimir, o tener ciertos efectos. Esta explicación se expone a parecer demasiado simplista, cuando precisamente el acontecimiento a analizar comporta un gran número de parámetros... como ejemplo típico tenemos el ser humano, ante el cual vemos que no se puede adoptar una actitud reduccionista, prueba de ello son las enfermedades psicosomáticas. Aún más, las ideas preconcebidas, y justificadas mediante el proceso deductivo (hacer corresponder lo que uno ve con lo que uno sabe), ciertamente encierran a la persona, la enajenan, la clasifican e impide una visión global del ser humano.

A nuestro parecer, este abordaje causal y deductivo de considerar al hombre es inseparable de la visión "científica" y analítica, pero este condiciona a la persona, es de alguna

manera determinista e incompleto. Entendámonos bien: no se trata, ni mucho menos, de rechazar de golpe y sin más el proceso científico y analítico. Después de todo, un problema diagnosticado como renal, por ejemplo, pide y exige abordarlo primeramente de forma macroscópica para ir a la microscópica, de lo general a lo particular. Esto es válido y pensamos seguirá siendo válido, pero pensamos es parcial.
Nos parece por tanto esencial, que todo el proceso médico y terapéutico de una persona enferma no se resuma solamente a este tipo de investigación, (causaefecto)sino que debe, a nuestro parecer, ser completado por una comprensión global, holística, de lo que vive el enfermo; por tanto que esté completado por un proceso inductivo, no causal, en el que se tenga en cuenta el encuentro relacional con lo que vive, siente, piensa... la persona, en tanto que acontecimientoúnico. Nuestro objetivo no es pararnos en hacer una enumeración de los límites del proceso científico-analítico basado en las relaciones de causalidad. Permítasenos, a título de ejemplo, citar dos campos, en donde la concepción monolítica antigua, que ha evolucionado muy poco, nos parece no tener mucho que ofrecernos si continua a reinar en solitario:

- las enfermedades psicosomáticas, en las cuales la visión del órgano enfermo es indisociable con la enfermedad de la persona concebida como un todo.

- las enfermedades que tocan la esfera psíquica de la relación (relación con el mundo o enfermedades llamadas mentales), que son, por otra parte, como imposibles de comprender de manera causal, pues estas enfermedades representan precisamente una negativa a ser comprendidas.

c) Un caso particular, o la puesta en evidencia de los límites del método analítico en las "afecciones -límites".

Se trata de una patología cada vez más frecuente. Tenemos que confesar que nuestra formación basada en una filosofía analítica deductiva, nos prepara bastante mal, a mi parecer, para una relación terapéutica con la persona enferma. La idea de que la toma de conciencia del material inconsciente "cura" el síntoma,(causa, luego efecto) no la podemos aplicar de ninguna manera a un "paciente" que concibe la relación terapéutica como un "deseo de recibir" y no como un deseo de rechazar los síntomas negativos. Esta última concepción que realiza con frecuencia el enfermero/a es a menudo una reducción errónea de la demanda del paciente. Pero a este deseo de recibir de la persona "borderline", se opone su miedo a ser encerrada en su mismo "recibir", de aquí la profunda ambivalencia y cautividad en la que viven estas personas. En efecto, la idea de un modelo preestablecido, en el cual el enfermo es por una parte comprendido ( en el sentido amplio de la palabra) y por otra 
"encerrado", si bien es a menudo el modo de relación generalmente propuesto por el paciente, es también antiterapéutico, y desmotivante para la enfermera, pues el poder que ejerce la enfermera así idealizada no hace más que reforzar el modo de relación patológico y ambivalente del paciente que siente deseo y miedo a ser amado por una madre-objeto idealizada ( una madre que no es en la realidad percibida siempre como persona por el paciente).

En estas condiciones el paciente no tiene necesidad del amor alienante de la madre idealizada, sino más bien del amor diferenciado y adaptado, y por tanto sin peligro, de su propia madre simbólica. Eso implica, una doble relación: por una parte, que la enfermera ponga el acento particularmente sobre la percepción "verdadera" y auténtica de la persona del otro; y por otra, que se centre en su realidad.

\section{d) "Afecciones-límites" y cuidados hospitala-} rios.

Una cierta semejanza de comportamiento parece existir, momentáneamente en el paciente hospitalizado, formando parte de la ausencia de libertad en la afecciones-límites. Estas dos condiciones muestran por parte del paciente, por una parte, el deseo de regresar (hablamos en términos de la psicología dinámica), justificado o no, y por otra, una necesidad ambivalente de idealizar a la enfermera y de curarse, sintiendo como una injusticia/injuria que la "naturaleza" ha cometido hacia él y de la que no puede escapar. Esta semejanza nos parece fecunda en cuanto a la visión de los límites de los esquemas tradicionales, y en cuanto a la manera de salir de ellos. Nosotros nos permitiremos pues, por razones prácticas, considerar metodológicamente estas dos condiciones como un todo, cuyos elementos pueden mutuamente esclarecerse.

.. y visto lo que precede, ¿ qué podemos esperar de la fenomenología?

\section{Breve abordaje de la fenomenología.}

Esta está basada en el pensamiento matemático de Husserl, y se opone al naturalismo, que trata el psiquismo humano como un objeto físico, mientras que "nosotros explicamos"su naturaleza y tratamos de comprender la vida psíquica" ( W.Dilthey). Hay una vuelta a las cosas mismas, de las cuales podemos discernir el sentido y la esencia, no de una manera mís- tica, sino más bien perceptible por "transparencia", esencia que es una intuición del sentido (de la misma manera que hay una "esencia de un círculo" en el circulo deformado que pinta un niño). La necesidad de esta esencia (su carácter idéntico en todos los casos) se opone a su facticidad que es la parte cambiante de cada manifestación. Este mundo de las esencias existe en la conciencia del sujeto, y no existe más conciencia que la dirigida hacia un objeto. De la misma manera que un objeto no existe más que para un sujeto. La visión del mundo está continuamente dominada por un análisis del dinamismo del espíritu dando su sentido a los objetos del mundo. La esencia de un objeto implica el punto de mira de su significación, que es inseparable del objeto mismo. De aquí el peligro de confundir la esencia del fenómeno con el estado de la conciencia propia del sujeto. El proceso fenomenológico consiste pues en una extracción de la esencia "trascendente" del contenido de la conciencia. Aquí se percibe la noción de la totalidad invariante tal como la definirá la teoría de la Gestalt.

Un capítulo importante de la fenomenología es su metodología de la comprensión. Esta, estima que lo humano está por comprender, que hay un "saber sobre el objeto" (intencionalidad temática) que se esfuerza, sin cesar y en vano, por reconquistar el punto de vista del objeto a través de los hechos (intencionalidad operante). La actitud de la fenomenología ante el ser humano está basada en la reflexión y en la inducción, y no en lo analítico (el psicoanálisis reposa sobre una visión causal de las interacciones que discierne o cree discernir). La unidad del YO es por otra parte comprendida como la unidad de la historia de la persona. La fenomenología nos permite situarnos frente al otro (al tú) con un tipo de comprensión de orden superior, por una intuición del "ser-hombre".(Binswanger). En materia del psiquismo humano, lo vivido por la persona no será a veces inmediatamente perceptible desde el exterior. Serán pues necesarios datos objetivables, afín de descubrir qué dimensión fundamental del "ser-hombre" (por la cual es definida el existente humano) está tocada por la enfermedad. Si nosotros añadimos que eso será determinado no directamente por el cuadro clínico, sino por la intuición de la enfermera, nosotros queremos bien precisar que se trata de una intuición de la esencia del fenómeno patológico que trasciende sus manifestaciones particulares. Eso es posible, pues la enfermedad, a pesar de la modificación de una 
dimensión fundamental de la existencia humana, permanece un fenómeno humano (Husserl).

Pasar a través de una relación terapéutica del YO (Ego) a la dimensión del otro pone en juego una "intersubjetividad transcendental", que se sitúa en un "intermundo que mediatiza los intercambios" (Merleau-Ponty), intercambios que implican comprehensión si la distancia relacional existe. Con esto queremos decir que a través de la relación llegamos, o podemos llegar, al "fenómenon", lo que aparece a la luz, sin llegar a deformarlo por unos modos sesgados de observación. Dicho de otra manera, el cuerpo es la "sede" de movimientos que es preciso comprender no solamente como consecuencias de las emociones, inhibidas o no, pues toda relación de causalidad deforma el abordaje de la realidad.

En las perturbaciones, llamadas funcionales, podíamos decir, "el paciente como que no vive", pues "tomado" por sus emociones, tiende a somatizar. Hay un deslizamiento hacia la somatización de las posibilidades vitales. Añadamos que la emoción violenta implica un encogimiento del campo vital, de la conciencia de existir, de ese "Daseim"(Heidegger) que es el lugar de paso para la realización de nuestras relaciones con el mundo.

\section{sión. \\ Aportes prácticos de la fenomenología y discu- \\ 1.- Aportes prácticos. "Todo esto es bello si es útil".}

Uno podría preguntarse: de todo lo que acabamos de decir ¿qué consecuencias prácticas puede sacar el enfermero/a?

\section{Citemos algunas consecuencias prácticas:}

- Una actitud terapéutica centrada: sobre la verdadera escucha (escucha activa según la psicoterapia fenomenológica rogeriana), sobre la apertura a lo que es verdaderamente el otro, dejándose interrogar por su presencia.

- El hecho de no buscar a todo precio una categoría nosológica en la cual encerrar al paciente.

- Salir de las explicaciones causales simplistas y reduccionistas (en el sentido peyorativo de los términos).

Interrogarse no sobre el órgano que se encuen- tra enfermo, sino de preguntarse QUIEN sufre, qué está verdaderamente viviendo el paciente. (Aquí tendríamos la respuesta al título del trabajo: en contraposición al método deductivo (causa-efecto), la fenomenología, a través de la intuición (proceso inductivo) nos conduce a aquello que verdaderamente interesa a Husserl: lo eidético, la esencia del individuo, lo que éste es con todo lo que esto conlleva.

- Tener presente, en la conciencia, la importancia de la búsqueda de la unidad del paciente, de su historia igualmente.

- Permanecer permeable a su intuición de "estar en el mundo", a su "esencia" y a la intencionalidad que da a su persona.

\section{Los límites del proceso fenomenológico. o "El} fin de un bonito sueño"

Este proceso nos parece tener también sus defectos y sus cualidades. Teniendo en cuenta una cierta objetividad, la enfermera en su observación del paciente, a veces tiende a suprimir toda interacción, nos parece que la enfermera no siempre toma conciencia de la importancia de su relación, ni domina, por otra parte, la importancia del juego de las interacciones, de la transferencia y contra-transferencia, en el sentido del que nos habla S. Freud. A fuerza de querer ver y alcanzar, el verdadero "estar en el mundo" del paciente, este método fenomenológico nos parece quedarse un tanto pobre, pues para muchos de nuestros pacientes la comunicación pasa no por el ser, sino por el "hacer en el mundo", o el "tener en el mundo", y la facticidad es tal que obnubila toda relación terapéutica. Fácilmente se tiene la impresión de que esta actitud no inmuniza completamente al terapeuta contra la manipulación... Se desearía que la fenomenología nos suministrase una herramienta más cualificada del estudio de la relación terapéutica, así como del poder de motivación y de cuestionamiento de la enfermera-misma.

Por otra parte, si los aportes de la fenomenología nos parecen esencialmente positivos, esto no es un obstáculo par a que nosotros tratemos de hacer observar ciertas reservas sobre el plan del subjetivismo, que nos parece estar muchas veces presente. Estimamos que a pesar de ciertas adaptaciones de ponerlo al día, aún permanece fuerte la tentación para el fenomenólogo de creer que el proceso fenomenológico de intuición da acceso a lo vivido del otro (personal, histórico). 
Sin querer hacer un reproche a la fenomenología, uno de sus límites está, a nuestro parecer, en la cuestión de la relación terapéutica que hace referencia a la vivencia de lo espiritual. Este límite nos parece particularmente manifiesto debido a que el aspecto fenomenológicamente "transcendente" de la intuición oculta toda trascendencia de orden superior y esto de forma reductora sobre todo en su primer momento (primum movens) de retorno a las cosas.

\section{Discusión. ¿Y por qué no?}

El psicoanálisis representa la primera corriente que ha puesto a disposición del enfermero/a un instrumento verdaderamente útil de investigación de la relación y de lo vivido inconscientemente. Sus límites también son percibidos claramente en la situación actual de la clínica, cuando la enfermera se confronta al paciente, no a causa de la dialéctica pulsional consciente-inconsciente, sino a un nivel no neurótico, donde el paciente no presenta pues una demanda inconsciente de "querer desprenderse" de un síntoma molesto, sino más bien de querer recibir una relación de dependencia o incluso de fusión. Por otra parte, la actitud fenomenológica nos parece representar una vuelta hacia una relación más auténtica, más verdadera, más útil, ( lógicamente si esta se realiza bien ) y por tanto menos inclinada a caer en una relación de "poder" y desembarazada de todo subjetivismo. Hemos de decir, que todo el debate sobre la manera en la que acogemos o recibimos al otro al tú, nos parece más útil (y menos doctrinal) si este se sitúa en perspectiva de la identidad de cada uno, y que está por descubrir. Esta identidad nos parece una noción mas inmutable y menos dependiente de las circunstancias y de la "facticidad" (por hablar en términos de la fenomenología). La negativa de esta identidad, consciente o no, entraña la imposibilidad de inversión sobre el yo, cuya huida en la psicosis podría representar una de las consecuencias posibles. Por otra parte, la noción fenomenológica de intencionalidad, con sus variantes, aplicada al ser humano, nos parece ceder paso ante el de finalidad.

\section{4. ¿Qué aplicación concreta se puede hacer en los cuidados de enfermería?}

La plataforma sobre la que nos hemos situado, al principio de este trabajo, para situar los cuidados de enfermería en un contexto de buscar recibir, y el contexto terapéutico general de estas líneas, nos parece indicar de forma bastante clara lo que podemos esperar de este proceso fenomenológico con vistas a una aplicación concreta, aplicación que pienso está todavía en muchos aspectos por construir.

A modo de ejemplo, señalemos principalmente la importancia para los enfermeros/as de percibir la enfermedad institucional como un tiempo en la vida de la persona que se inserta en toda una trayectoria de vida, la cual en el fondo se nos escapa. Durante este tiempo se le pone un diagnóstico más o menos global con respecto a un síndrome, pero nos olvidamos que el aspecto holístico del ser humano es algo más que el conjunto de síntomas que definen a la persona enferma en un momento dado de su historia.

\section{Conclusión.}

Estamos de acuerdo en que ninguna corriente filosófica puede ser idealizada. Sin embargo, nos parece importante el que la fenomenología cuestione la utilidad de los métodos tradicionales. Nos parece esencial la vuelta a la verdadera interrogación de la identidad del otro. La evolución probable de las diversas patologías hace que el instrumento que propone la fenomenología de una nueva consistencia a la terapia, de manera especial a aquellos que sufren de enfermedades psicosomáticas o de estados-límites. Por otra parte será muy útil poner en marcha la aplicación fenomenológica pues nos ofrece una visión renovada del otro y de la relación con su cuerpo, y todo esto sabiendo que no es la panacea, sino un paso más en la comprehensión del misterio de la persona humana cuando esta es visitada por la enfermedad. Nos preguntamos:¿cómo actúa en el ser humano la relación de ayuda terapéutica? Esta pregunta nos parece que puede tener una doble realidad. Por una parte la de ¿cómo es posible, en general, que la relación de ayuda terapéutica puede actuar en el ser humano? Y por otra ¿cómo puede actuar de esta o de la otra manera? Bajo el primer aspecto, se trata de la cuestión científica, en sentido radical, la del fundamento: ¿cuál es en general la condición de la posibilidad de la acción terapéutica de la relación? En segundo lugar, se trata de una cuestión técnica, que concierne el arte del saber de la enfermera. Es esencial distinguir estas dos cuestiones, y sobretodo, contrariamente a la tendencia de nuestra civilización técnica, de no confundir la primera con la segunda. En 
la perspectiva de la primera cuestión, la relación terapéutica se presenta cono una forma de encuentro de un ser humano con otro ser humano, encuentro que comporta siempre un momento de relación (explicación) con el otro. La condición de posibilidad de la acción terapéutica reside en la dimensión comunicativa, en tanto que dimensión esencial del ser humano. En la relación terapéutica, propiamente dicha, entre el enfermero/a y el paciente, esta dimensión sufre una triple reducción: el enfermo no es considerado como "partenaire", sino considerado desde el punto de vista de su mal funcionamiento físico o psíquico, y por tanto el enfermero/a desaparece en tanto que persona, detrás de su función de cuidador, la relación se hace en sentido único, ni se hace más que desde el punto de vista de una acción del enfermero/a sobre el paciente; esta relación no puede ser pues considerada plenamente como una relación de hombre a hombre, sino como un cuidado limitado a una causa precisa. No confundir la primera cuestión con la segunda, significa también que toda intervención terapéutica se sitúa en el marco de un encuentro de la persona con la persona, la cual no puede ni ignorar ni descuidar. Esta intervención supone en efecto, con el fin de que tenga sentido y valor, la confianza que el enfermo concede al enfermero/a, y la confianza que el enfermero/a concede al paciente. Hay pues una dialéctica necesaria en toda relación terapéutica entre la dimensión comunicativa-personal y la dimensión objetivante-funcional. Y esta dialéctica tiene como consecuencia que no hay una intervención terapéutica ya hecha, estándar, sino que esta hay que "inventarla", total o en parte en cada situación.

Una relación terapéutica debe ser "inventada cada vez", porque el ser humano que cuidamos no es un objeto al que reparamos sin más, sino un ser que sufre y que tiene necesidad de ayuda para madurar y volver a encontrar el sentido de su vida o de su voca- ción en el mundo. Por esta razón entendemos el que un elemento muy importante de la relación terapéutica o de ayuda sea justamente la exploración de la historia o de la biografía interior del ser enfermo, con la finalidad de desatar los bloqueos que están restringiendo sus posibilidades de existir de una manera libre y personal.

En numerosos casos, este intentar disminuir los bloqueos significa para el enfermo el tratar de saciar ciertas posibilidades corporales (su voz, por ejemplo en casos de afonía). Pero ¿cómo es posible de volver a encontrar una posibilidad corporal perdida?. Para darnos cuenta de esto es necesario comprender lo que es el cuerpo ( en tanto que cuerpo vivido) y principalmente de hacer justicia de las falsas evidencias del llamado paralelismo psico-físico. La verdadera comprensión de lo que es el cuerpo se decide en la comprensión del lenguaje del cuerpo. A partir de aquí lo que llamamos síntoma toma una significación que muchas veces no tiene nada que ver con el pensamiento que está debajo de las explicaciones mecanicistas y causales. Concluiremos contestando a la pregunta objeto de este trabajo:¿Qué nos aporta la fenomenología a la relación enfermera -paciente? La fundamentación de nuestros cuidados holístico y ayudarnos a encontrar nuestra identidad como enfermeros/as.

\section{BIBLIOGRAFÍA}

Husserl, E. (1982) La idea de la fenomenología. Fonde de Cultura Económica Madrid.

Hirschberger (1965) Historia de la Filosofía II. Herder, Barcelona 